\title{
Experimental endogenous septicaemia caused by Klebsiella pneumoniae and Escherichia coli in mice
}

\author{
Y. HIRAKATA*, N. FURUYA, T. MATSUMOTO, K. TATEDA and K. YAMAGUCHI \\ Department of Microbiology, Toho University School of Medicine, Tokyo 143, Japan
}

\begin{abstract}
Multi-resistant Klebsiella pneumoniae have recently occured in several nosocomial outbreaks of septicaemia. An animal model resembling the pathophysiology of these infections in man would be very useful. A new model of endogenous septicaemia caused by $K$. pneumoniae and Escherichia coli strains in mice has been established. The mortality rate of conventional ddY mice given cyclophosphamide (CY) or fluorouracil (5-FU), each $200 \mathrm{mg} / \mathrm{kg}$ intraperitoneally, every other day was 70 and $100 \%$, respectively. Pseudomonas aeruginosa septicaemia was observed in all dead mice treated with CY, whereas Enterobacteriaceae, including $K$. pneumoniae, were isolated from $90 \%$ of mice given 5-FU. Specific-pathogen-free mice, decontaminated with ampicillin and ceftazidime, were given multi-resistant $K$. pneumoniae CF504, CF514 or E. coli CF604, or CF614 carrying CAZ-1/TEM-5 plasmid by oral inoculation. Subsequent dosing with 5-FU induced lethal septicaemia caused by the inoculated strains in most of these mice, whereas CY did not regularly induce septicaemia. This model with $5-\mathrm{FU}$ is considered to resemble closely the situation observed in man and to be beneficial for investigating pathophysiology and therapeutic strategies.
\end{abstract}

\section{Introduction}

Recently, several nosocomial outbreaks of infections including septicaemia caused by Klebsiella pneumoniae resistant to many antibiotics have been reported [1-3]. Moreover, $10-30 \%$ of these strains produced new extended-spectrum $\beta$-lactamases, cefotaximase or ceftazidimase, which confer resistance to broad-spectrum cephalosporins [4,5]. Epidemiological investigations have revealed that the reservior for the $K$. pneumoniae strains lies in the gastrointestinal tract of the patients $[2,6]$. To investigate the prophylaxis and treatment of septicaemia caused by endogenous $K$. pneumoniae, an animal model resembling the pathophysiology of septicaemia in man has been set up.

The present study sets out to compare the influence of cyclophosphamide and fluorouracil on the frequency of occurrence of endogenous septicaemia and differences in causative organisms in mice, with a new model of endogenous septicaemia caused by specific $K$. pneumoniae strains; decontamination of the mice with ampicillin and ceftazidime was followed by oral

Received 5 May 1995; accepted 18 Aug. 1995.

* Present address and address for correspondence: Department of Laboratory Medicine, Nagasaki, University School of Medicine, Nagasaki 852, Japan. inoculation of $K$. pneumoniae resistant to these antibiotics.

\section{Materials and methods}

Reagents

Sodium ampicillin (AMP, Viccillin; Meiji Seika Kaisha, Ltd, Tokyo, Japan), ceftazidime (CAZ, Modacin; Nippon Glaxo Co., Ltd, Tokyo, Japan), cyclophosphamide (CY, Endoxan; Shionogi Co. Ltd, Osaka, Japan), fluorouracil (5-FU, 5-FU Kyowa; Kyowa Co. Ltd, Tokyo, Japan) were purchased from commercial sources. Imipenem (IPM) was a gift from Banyu Pharmaceutical Co. Ltd, Tokyo, Japan.

\section{Animals}

Conventional and specific-pathogen-free (SPF) male ddY mice (Japan S.L.C. Co. Ltd, Shizuoka, Japan) weighing $20-24 \mathrm{~g}$ were used in the experiments. Mice were fed a sterile diet and were given sterile distilled water except during the period of oral administration of the bacteria.

\section{Bacterial strains and media}

Multi-resistant K. pneumoniae CF504 and CF514 [7], their transconjugants Escherichia coli CF604 and 
CF614 [8], and cured K. pneumoniae CF504 [9] were kindly provided by Dr A. Darfeuille-Michaud, Laboratoire de Bacteriologie, Facultes de Pharmacie et Medecine, France. Multi-resistant strains, CF504 and CF514, were isolated from clinical blood samples and were first studied for a new expanded-spectrum $\beta$ lactamase plasmid, CAZ-1/TEM-5 [4]. The bacteria were grown on Trypticase Soy Agar (BBL Microbiology Systems, Cockeysville, MD, USA) at $37^{\circ} \mathrm{C}$ for $18 \mathrm{~h}$. They were then suspended in sterile saline $0.45 \%$ and the concentration was adjusted to $10^{7} \mathrm{cfu} / \mathrm{ml}$ based on the optical density of the cell suspension as determined by spectrophotometry (UVIDEC-40; Jasco, Tokyo, Japan).

\section{In-vitro susceptibility}

Minimum inhibitory concentrations (MICs) of antibiotics were determined by a microbroth dilution method [10] in Muller-Hinton Broth (Difco Laboratories, Detroit, MI, USA) supplemented with calcium $25 \mathrm{mg} / \mathrm{L}$ and magnesium $12.5 \mathrm{mg} / \mathrm{L}$. Bacteria were inoculated at a final concentration of $5.0 \times 10^{5} \mathrm{cfu} / \mathrm{ml}$.

\section{Influence of $C Y$ and 5-FU on the occurrence of endogenous septicaemia}

Endogenous septicaemia was induced as described previously [11], with some modifications. Conventional mice were given AMP $200 \mathrm{mg} / \mathrm{kg} /$ day intraperitoneally until day 15. These mice were also given CY $200 \mathrm{mg} /$ $\mathrm{kg}$ /day or the same dose of 5-FU intraperitoneally every other day from day 1 . Post-mortem cardiac puncture for blood culture was performed immediately on all mice that died. All samples were cultured and the isolates were identified as described previously [11].

\section{Establishment of endogenous $K$. pneumoniae and E. coli septicaemia model}

SPF mice were given CAZ $200 \mathrm{mg} / \mathrm{kg}$ and AMP $200 \mathrm{mg} / \mathrm{kg}$ daily until the end of experiments for bowel decontamination. Faecal specimens were collected every other day before dosings, diluted and cultured on modified Drigalski agar with and without CAZ $5 \mathrm{mg} / 1$. Mice were fed multi-resistant $K$. pneumoniae CF504 or CF514, E. coli CF604 or CF614, or cured $K$. pneumoniae CF504 in their drinking water between days 5 and 8. Cyclophosphamide or 5-FU were administered on days $9,11,13,15$ and 17. Mice were monitored until day 20 and post-mortem cardiac punctures for blood culture were performed as described above. Samples were cultured on modified Drigalski agar with and without CAZ.

\section{Statistical analysis}

The $\chi^{2}$ test and Student's $t$ test were used to compare survival rates and means, respectively. A p value level of 0.05 was considered to be statistically significant.

\section{Results}

Influence of $\mathrm{CY}$ and 5-FU on the occurrence of endogenous septicaemia

As shown in Fig. 1, mice treated with $\mathrm{CY}$ began to die on day 6 and the final mortality rate was $70 \%$. The mice treated with 5-FU began to die later than those treated with CY, but by the end of the experiment all mice had died. $P$. aeruginosa strains were isolated from seven mice treated with $C Y$ and one mouse treated with 5-FU. On the other hand, Enterobacteriaceae were isolated from nine mice treated with 5-FU. The microorganisms isolated from these mice are listed in Table 1. Among the mice killed during the experiment, Morganella morganii was isolated from two of three animals treated with CY.

\section{Establishment of $K$. pneumoniae and E. coli septicaemia model}

As shown in Table 1, septicaemia caused by Enterobacteriaceae, including $K$. pneumoniae, occurred frequently in conventional mice treated with 5-FU. Enterobacteriaceae, including $K$. pneumoniae and $E$. coli, isolated from conventional mice with endogenous septicaemia were sensitive to ceftazidime (MICs $<4.0 \mathrm{mg} / \mathrm{L}$ ). Enterobacteriaceae isolated from faecal specimens of SPF mice were also sensitive to the antibiotic.

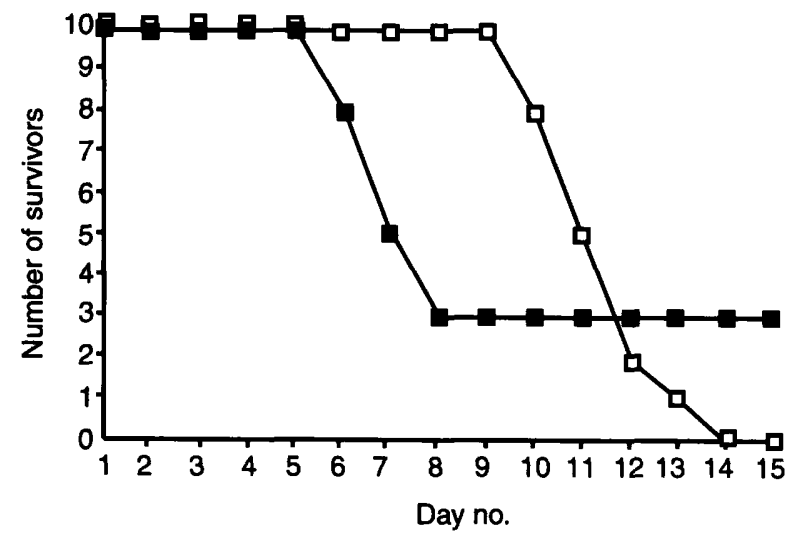

Fig. 1. Survival kinetics of conventional mice treated with CY or 5-FU. Conventional mice were given AMP $200 \mathrm{mg} / \mathrm{kg} /$ day intraperitoneally until day 15 and $\mathrm{CY}$ or $5-\mathrm{FU} 200 \mathrm{mg} / \mathrm{kg} /$ day intraperitoneally every other day from day 1 (10 mice/group); $\square$, CY-treated mice, $\square$, 5FU-treated mice.

Table 1. Micro-organisms isolated from cardiac blood of conventional mice treated with $\mathrm{CY}$ or 5-FU

\begin{tabular}{lcc}
\hline & \multicolumn{2}{c}{ Number of mice treated with } \\
\cline { 2 - 3 } Micro-organisms & CY & 5 -FU \\
\hline$P$. aeruginosa & 7 & 1 \\
$K$. pneumoniae & 0 & 4 \\
$K$. oxytoca & 0 & 3 \\
Ent. cloacae & 0 & 2 \\
$M$. morganii & $2^{*}$ & 0 \\
None & $1^{*}$ & 0 \\
\hline
\end{tabular}

* Mice that were killed at the end of the experiments. 
Table 2 summarises the MICs of AMP, CAZ and IPM against the bacterial strains used in this study. All strains were resistant to AMP and sensitive to IPM. $K$. pneumoniae CF504 and CF514, and E. coli CF604 and CF614 were resistant to CAZ, whereas cured $K$. pneumoniae CF504 was sensitive to CAZ.

SPF mice had (4.59 SEM 2.33) $\times 10^{6} \mathrm{cfu} / \mathrm{g}$ of CAZ sensitive lactose-fermenting gram-negative rods (GNR) in their faeces before the administration of CAZ and AMP. After bowel decontamination with antibiotics, lactose-fermenting GNR in faecal specimens completely disappeared by day 3 (limit for detection was $20 \mathrm{cfu} / \mathrm{g}$ ). Therefore, mice were given multi-resistant $K$. pneumoniae, $E$. coli or cured $K$. pneumoniae CF504 on days 5-8. Fig. 2 shows the kinetics of lactose-fermenting GNR sensitive or resistant to CAZ in the faecal specimens from mice after bowel decontamination and oral inoculation of $K$. pneumoniae strains. CAZ-resistant lactose-fermenting GNR were constantly detected in the stools of mice at concentrations $>10^{7} \mathrm{cfu} / \mathrm{g}$ until day 15 when mice were given $K$. pneumoniae CF504 or CF514. Stool samples could not be obtained after day 15 because of death or constipation of the mice. When mice were inoculated with the cured strain CF504, CAZ-sensitive

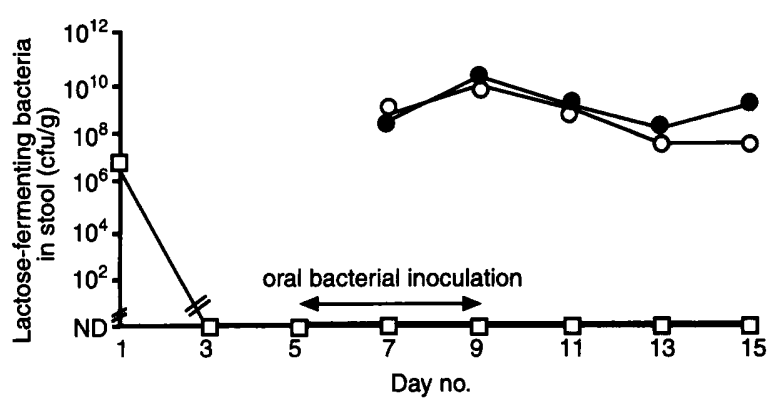

Fig. 2. Kinetics of CAZ-sensitive and CAZ-resistant lactose-fermenting GNR in stools of mice treated with CAZ and AMP and inoculated orally with $K$. pneumoniae CF504 and CF514. The results are expressed as the mean of values obtained for five mice in each group. $\square, \mathrm{CAZ}$ sensitive lactose-fermenting GNR; $O$, CAZ-resistant lactose-fermenting GNR in stools of mice given CF504; -, CAZ-resistant lactose-fermenting GNR in stools of mice given CF514. lactose-fermenting GNR were detected in their stools from day 7 to day 11 . However, the level was 100 -fold lower than that of CAZ-resistant lactose-fermenting GNR in mice given strains CF504 or CF514 ( $p<0.01$ between day 7 and 11), and decreased below the limit of detection by day 13 (data not shown).

As shown in Fig. 3, the mortality rates of mice fed $K$. pneumoniae CF504 and CF514 were 70 and $100 \%$, respectively, when they were treated with 5-FU, whereas the mortality rates of mice treated with $\mathrm{CY}$ were apparently lower than those of mice treated with 5 -FU $(30 \%$ versus $70 \%$ in CF504, $p<0.05 ; 10 \%$ versus $100 \%$ in CF514, $p<0.01$ ). Pure growths of CAZ-resistant $K$. pneumoniae were isolated from blood cultures of all dead mice. None of the mice fed cured K. pneumoniae CF504 and treated with CY or 5-FU died. The blood samples from these mice were sterile at the end of the experiments.

Fig. 4 shows the survival kinetics of mice fed $E$. coli CF604 or CF614 and treated with 5-FU. Mice began to die later those fed $K$. pneumoniae strains; however, by day 25 , all mice had died due to CAZ-resistant $E$. coli septicaemia.

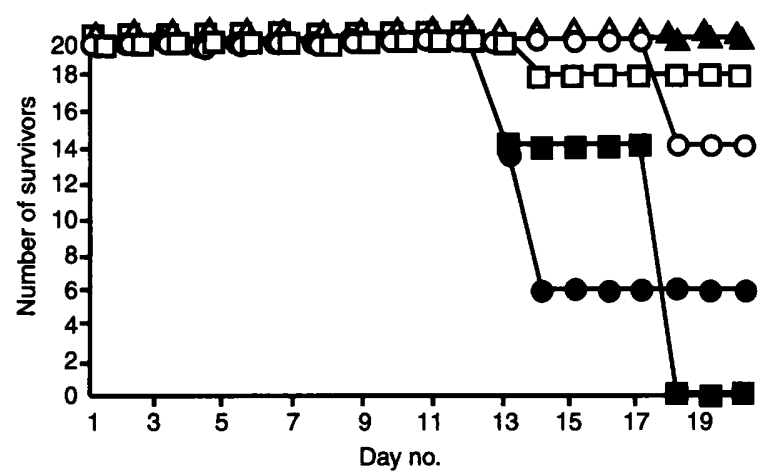

Fig. 3. Survival kinetics of mice treated with antibiotics, inoculated with $K$. pneumoniae strains and given $\mathrm{CY}$ or 5-FU (10 mice/group). O, CF504 and CY; 9 , CF504 and 5-FU; $\square$, CF514 and CY; $\square$, CF514 and 5-FU; $\triangle$, cured cF504 and CY; $\Delta$, cured CF504 and 5-FU. The mortality rates of mice given $K$. pneumoniae CF504 and CF514 and treated with 5-FU were significantly higher than those of mice treated with CY $(p<0.05$ and $p<0.01$, respectively).

Table 2. MICs of antibiotics against $K$. pneumoniae and $E$. coli strains used in this study

\begin{tabular}{|c|c|c|c|}
\hline \multirow[b]{2}{*}{ Strains } & \multicolumn{3}{|c|}{ MICs $(\mathrm{mg} / \mathrm{L})$} \\
\hline & Ampicillin & Ceftazidime & Imipenem \\
\hline \multicolumn{4}{|l|}{$K$. pneumoniae } \\
\hline CF504 & $>128$ & 32 & 0.125 \\
\hline CF514 & $>128$ & 16 & 0.125 \\
\hline Cured CF504* & $>128$ & 0.5 & 0.125 \\
\hline \multicolumn{4}{|c|}{ E. coli, transconjugants ${ }^{\dagger}$} \\
\hline CF604 & $>128$ & 32 & 0.125 \\
\hline CF614 & $>128$ & 32 & 0.125 \\
\hline
\end{tabular}

${ }^{*}$ CF504 strain cured of its 185 -kb $\mathrm{R}$ plasmid encoding resistance to broad-spectrum cephalosporins, streptomycin, kanamycin, neomycin, tetracycline and sulphonamide.

${ }^{\dagger}$ Transconjugants harbouring the $185-\mathrm{kb}$ R plasmid of the corresponding $K$. pneumoniae strains. 


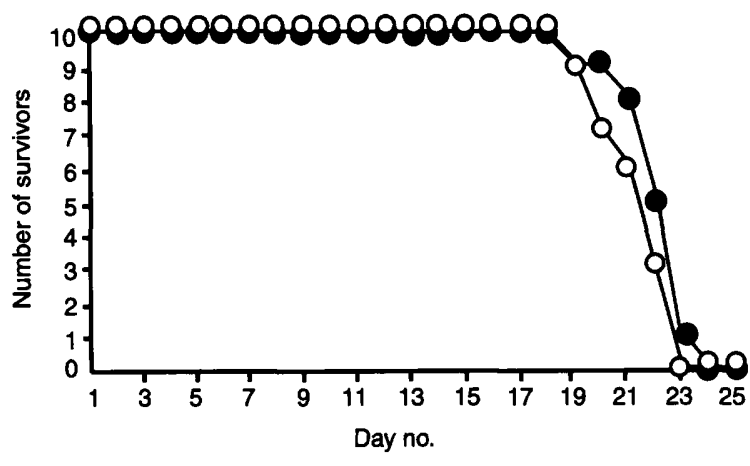

Fig. 4. Survival kinetics of mice given E. coli CF604 or CF614 and treated with 5-FU (10 mice/group). $O$, CF604; CF614.

\section{Discussion}

We have reported previously that $\mathrm{CY}$ often induces endogenous $P$. aeruginosa septicaemia in mice [11]. Moreover, it was possible to induce endogenous septicaemia with specific $P$. aeruginosa strains by administering CY and AMP to mice fed these organisms [12]. These animal models are useful for the investigation of the pathophysiology of endogenous septicaemia, the virulence factors of $P$. aeruginos $a$ and therapeutic strategies [12-15].

Enterobacteriaceae such as $K$. pneumoniae and $E$. coli cause portal septicaemia only in CY-treated mice because they are easily cleared from blood by the reticulo-endothelial system, especially by bacterial association with Kupffer cells in the liver [11,16]. Therefore, suitable immunosuppressive agents other than CY are required to establish a model of endogenous septicaemia caused by these organisms. Additionally, the use of germ-free or gnotobiotic mice, or bowel decontamination may be necessary to induce septicaemia with a specific strain given orally because there are many resident Enterobacteriaceae in the intestinal tract. Moreover, because most nosocomial infections, including septicaemia, occur after the use of broad-spectrum antibiotics, a model based on bowel decontamination by administration of antibiotics may closely resemble the situation observed in man.

As shown in this study, 5-FU frequently induced septicaemia caused by Enterobacteriaceae in conventional mice. This immunosuppressive agent also induced $K$. pneumoniae and $E$. coli endogenous septicaemia caused by specific strains after bowel decontamination and oral inoculation of multi-resistant strains in mice. The new model described in this study may be useful to investigate the pathophysiology of endogenous septicaemia and, the virulence factors of causative organisms. It is also possible to adopt this model for therapeutic and prophylactic strategies with carbapenems or immunopotentiating agents.

\section{References}

1. Brun-Buisson C, Legrand $\mathrm{P}$, Philippon A, Montravers F, Ansquer M, Duval J. Transferable enzymatic resistance to third-generation cephalosporins during nosocomial outbreak of multiresistant Klebsiella pneumoniae. Lancet 1987; 2: 302306.

2. De Champs C, Sauvant MP, Chanal C et al. Prospective survey of colonization and infection caused by expanded-spectrumbeta-lactamase-producing members of the family Enterobacteriaceae in an intensive care unit. $J$ Clin Microbiol 1989; 27: 2887-2890.

3. Sirot J, Chanal C, Petit A, Sirot D, Labia R, Getbaud G. Klebsiella pneumoniae and other enterobacteriaceae producing novel plasmid-mediated $\beta$-lactamases markedly active against third-generation cephalosporins: epidemiologic studies. Rev Infect Dis 1988; 10: 850-859.

4. Petit A, Sirot DL, Chanal CM et al. Novel plasmid-mediated beta-lactamase in clinical isolates of Klebsiella pneumoniae more resistant to ceftazidime than to other broad-spectrum cephalosporins. Antimicrob Agents Chemother 1988; 32: 626-630.

5. Sirot D, Sirot J, Labia R et al. Transferable resistance to thirdgeneration cephalosporins in clinical isolates of Klebsiella pneumoniae: identification of CTX-1, a novel beta-lactamase. $J$ Antimicrob Chemother 1987; 20: 323-334.

6. Markowitz SM, Veazey JM, Macrina FL, Mayhall CG, Lamb VA. Sequential outbreaks of infection due to Klebsiella pneumoniae in a neonatal intensive care unit: implication of a conjugative $\mathrm{R}$ plasmid. J Infect Dis 1980; 142: 106-112.

7. Hirakata $\mathrm{Y}$, Tomono $\mathrm{K}$, Tateda $\mathrm{K}$ et al. Role of bacterial association with Kupffer cells in occurrence of endogenous systemic bacteremia. Infect Immun 1991; 59: 289-294.

8. Hirakata $\mathrm{Y}$, Kaku M, Tomono $\mathrm{K}$ et al. Efficacy of erythromycin lactobionate for treating Pseudomonas aeruginosa bacteremia in mice. Antimicrob Agents Chemother 1992; 36: 1198-1203.

9. Darfeuille-Michaud A, Jallat C, Aubel D et al. R-plasmidencoded adhesive factor in Klebsiella pneumoniae strains responsible for human nosocomial infections. Infect Immun 1992; 60: 44-55.

10. Lesage DD, Gerbaud GR, Chabbert YA. Carte genetique et structure chez Escherichia coli K12 d'un plasmid de resistance isole de Salmonella ordonez. Ann Microbiol 1975; 126A: 435-438.

11. Bouanchaud DH, Scavizzi MR, Chabbert YA. Elimination by ethidium bromide of antibiotic resistance in enterobacteria and staphylococci. J Gen Microbiol 1968; 54: 417-425.

12. Sahm DF, Washington JA. Antibacterial susceptibility tests: dilution methods. In: Balows A, Hausler WJ, Herrmann KL, Isenberg HD, Shadomy HJ (eds) Manual of clinical microbiology, 5th edn. Washington, DC, American Society for Microbiology. 1991: 1105-1116.

13. Hirakata $\mathrm{Y}$, Furuya N, Tateda K, Kaku M, Yamaguchi K. In vivo production of exotoxin $\mathrm{A}$ and its role in endogenous Pseudomonas aeruginosa septicemia in mice. Infect Immun 1993; 61: 2468-2473.

14. Furuya N, Hirakata $\mathrm{Y}$, Tomono $\mathrm{K}$ et al. Mortality rates amongst mice with endogenous septicaemia caused by Pseudomonas aeruginosa isolates from various clinical sources. J Med Microbiol 1993; 39: 141-146.

15. Matsumoto $\mathrm{T}$, Kaku M, Furuya $\mathrm{N}$ et al. Amphotericin Binduced resistance to Pseudomonas aeruginosa infection in mice. J Antibiot Tokyo 1993; 46: 777-784.

16. Hirakata $\mathrm{Y}, \mathrm{Kaku} \mathrm{M}$, Furuya $\mathrm{N}$ et al. Effect of clearance of bacteria from the blood on the development of systemic bacteraemia in mice. $J$ Med Microbiol 1993; 38: 337-344. 